\title{
First-Principles Semiclassical Initial Value Representation Molecular Dynamics *
}

\author{
Michele Ceotto, ${ }^{1}$ Sule Atahan, ${ }^{2}$ Sangwoo Shim, ${ }^{2}$ Gian Franco Tantardini, ${ }^{1,3}$ and Alán Aspuru-Guzik ${ }^{2}$ \\ ${ }^{1}$ Dipartimento di Chimica Fisica ed Elettrochimica, Università di Milano, via Golgi 19, 20133 Milano, Italy ${ }^{\dagger}$ \\ ${ }^{2}$ Department of Chemistry and Chemical Biology, Harvard University, 12 Oxford Street, 02138, Cambridge, MA \\ ${ }^{3}$ Istituto CNR di Scienze e Tecnologie Molecolari, via Golgi 19, 20133 Milano, Italy
}

\begin{abstract}
In this work, we explore the use of the semiclassical initial value representation (SC-IVR) method with firstprinciples electronic structure approaches to carry out classical molecular dynamics. The proposed approach can extract the vibrational power spectrum of carbon dioxide from a single trajectory providing numerical results that agree with experiment and quantum calculations. The computational demands of the method are comparable to those of classical single-trajectory calculations, while describing uniquely quantum features such as the zeropoint energy and Fermi resonances. The method can also be used to identify symmetry properties of given vibrational peaks and investigate vibrational couplings by selected classical trajectories. The accuracy of the method degrades for the reproduction of anharmonic shifts for high-energy vibrational levels.
\end{abstract}

PACS numbers:

\section{INTRODUCTION}

Algorithms for the simulation of molecular dynamics belong to the fundamental toolset of modern theoretical chemical physics. Classical simulation methods are able to study systems with up to millions of particles but are unable to describe quantum effects such as tunelling and delocalization. Exact quantum mechanical methods are restricted to a few quantum particles, especially when pre-computed analytical potential energy surfaces (PES) are employed.

First-principles molecular dynamics (FPMD) algorithms have been introduced as an alternative to the pre-calculation of the PES. FPMD avoids any source of error originated from the fitting of the PES. This is particularly true for many degrees of freedom, where the fitting procedure might not represent the many-dimensional surface accurately. In this family of methods, the potential and its derivatives are calculated on-the-fly as the dynamical simulation progresses and are directly obtained from electronic structure calculations. In the Born-Oppenheimer molecular dynamics (BOMD) approach, the electronic structure calculations for a given simulation step are converged based on previous step information. This approach can lead to systematic energy drifts and several methods have been proposed to avoid this effect [1]. Alternatively, extended Lagrangian molecular dynamics approaches (ELMD) [2-5] involve the propagation of nuclear and electronic degrees of freedom simultaneously. The electronic degrees of freedom are assigned to classical variables that are propagated using classical equations of motion and these can be expanded in terms of plane waves [2], Gaussian functions [4] or real-space grids [5]. Usually ELMD propagation is computationally more efficient, however questions have raised on whether the resultant energy surface remains close to the actual Born-Oppenheimer one and about disturb-

${ }^{*}$ On the anniversary of the 100th year of foundation of the "Società Chimica Italiana" ing dependencies on the fictitious electronic masses $[4,6]$.

While the evaluation of the potential on-the-fly can be easily integrated with classical simulations, the delocalized nature of quantum mechanical propagation has led to the development of many alternative approaches for the simulation of quantum dynamics. For example, the path-integral centroid molecular dynamics approach [7] includes quantum nuclear effects employing an extended Lagrangian. Alternatively, in the variational multi-configuration Gaussian wavepacket method (vMCG) [8] the quantum wavepackets are represented by fixed-width Gaussian functions for which the potential is approximated to be locally harmonic. Other approaches introduce a mean field approximation and then update the dynamics in a time-dependent self-consistent fashion $[9,10]$.

Semiclassical molecular dynamics methods [11-20] are based on classical trajectories and therefore are amenable for carrying out on-the-fly calculation of the potential. The benefits of calculating the potential only when needed have been suggested by Heller and co-workers [20, 21]. In between formally exact quantum methods and classical dynamics, semi-classical methods include quantum effects approximately. Two representative semi-classical approaches are the coupled coherent states (CCS) technique [22] and the ab initio multiple spawing method (AIMS) algorithm [23]. In the CCS approach, several grids of coherent states are classically propagated and their trajectories can be derived from first principle dynamics. In AIMS, the nuclear wavefunction are spawned onto a multiple potential surface basis set. This set is made of adaptive time-dependent fixed-width Gaussian functions, which are generated by classical Newtonian dynamics.

\section{FIRST-PRINCIPLES SC-IVR}

In this work, we show how the semiclassical initial value representation (SC-IVR) [12] method can be coupled tightly and naturally, without any mayor change in formulation, with first principles electronic structure approaches to carry out classical molecular dynamics. We show how the method is 
able to reproduce approximately quantum effects such as the vibrational power spectra using a single, short classical trajectory using computational resources comparable to those employed in first-principles molecular dynamics calculations. Calculations employing multiple trajectories can in principle be more accurate (and more computational intense as well), but here we focus on analyzing the predictive power of single trajectory runs. Finally, we describe how different approaches can be used in conjunction with this method for studying the symmetry of the vibrational states either by arranging the initial conditions of the classical trajectory or by employing the symmetry of the coherent state basis.

In the SC-IVR method, the propagator in $F$ dimension is approximated by the phase space integral,

$$
\begin{aligned}
e^{-i \hat{H} t / \hbar}= & \frac{1}{(2 \pi \hbar)^{F}} \int d \mathbf{p}(0) \int d \mathbf{q}(0) C_{t}(\mathbf{p}(0), \mathbf{q}(0)) \\
& e^{i S_{t}(\mathbf{p}(0), \mathbf{q}(0)) / \hbar}|\mathbf{p}(t), \mathbf{q}(t)\rangle\langle\mathbf{p}(0), \mathbf{q}(0)|
\end{aligned}
$$

where $(\mathbf{p}(t), \mathbf{q}(t))$ are the set of classically-evolved phase space coordinates, $S_{t}$ is the classical action and $C_{t}$ is a preexponential factor. In the Heller-Herman-Kluk-Kay [19, 24] version of the SC-IVR, the prefactor involves mixed phase space derivatives

$$
\sqrt{\frac{1}{2}\left|\frac{\partial \mathbf{q}(t)}{\partial \mathbf{q}(0)}+\frac{\partial \mathbf{p}(t)}{\partial \mathbf{p}(0)}-i \hbar \gamma \frac{\partial \mathbf{q}(t)}{\partial \mathbf{p}(0)}+\frac{i}{\gamma \hbar} \frac{\partial \mathbf{p}(t)}{\partial \mathbf{q}(0)}\right|}=
$$

as well as a set of reference states $\langle\mathbf{q} \mid \mathbf{p}(t), \mathbf{q}(t)\rangle=$ $\prod_{i}\left(\gamma_{i} / \pi\right)^{F / 4} \exp \left[-\gamma_{\mathbf{i}} \cdot\left(q_{i}-q_{i}(t)\right) / 2+i p_{i}(t) \cdot\left(q_{i}-q_{i}(t)\right) / \hbar\right]$ of fixed width $\gamma_{i}$. For bound systems, the widths are usually chosen to match the widths of the harmonic oscillator approximation to the wave function at the global minimum and no significant dependency has been found under width variation [13]. By introducing a $2 F \times 2 F$ symplectic (monodromy) matrix $\mathbf{M}(t) \equiv \partial\left(\left(\mathbf{p}_{t}, \mathbf{q}_{t}\right) / \partial\left(\mathbf{p}_{0}, \mathbf{q}_{0}\right)\right)$, one can calculate the pre-factor of Eq. (2) from blocks of $F \times F$ size and monitor the accuracy of the classical approximate propagation by the deviation of its determinant from unity. Wang et al. suggested calculating the determinant of the positive-definite matrix $\mathbf{M}^{T} \mathbf{M}$ instead [25] and we monitored the same quantity for this work. The spectral density is obtained as a Fourier transform of the surviving probability[19]. The SC-IVR expression of the probability of survival for a phase-space reference state $|\chi\rangle=\left|p_{N}, q_{N}\right\rangle$ is

$$
\left\langle\chi\left|e^{-i \hat{H} t / \hbar}\right| \chi\right\rangle=\frac{1}{(2 \pi \hbar)^{F}} \int d \mathbf{p}(0) \int d \mathbf{q}(0) C_{t}(\mathbf{p}(0), \mathbf{q}(0)) e^{i S_{t}(\mathbf{p}(0), \mathbf{q}(0)) / \hbar}\langle\chi \mid \mathbf{p}(t), \mathbf{q}(t)\rangle\langle\mathbf{p}(0), \mathbf{q}(0) \mid \chi\rangle
$$

The phase-space integral of Eq. (3) is usually computed using Monte Carlo methods. If the simulation time is long enough, the phase space average can be well approximated by a time average integral. This idea has been suggested and im- plemented by Kaledin and Miller [26] to obtain the TA (Time Averaging [27]) SC-IVR approximation for the spectral density,

$$
\begin{aligned}
I(E) & =\frac{1}{(2 \pi \hbar)^{F}} \int d \mathbf{p}(0) \int d \mathbf{q}(0) \frac{\operatorname{Re}}{\pi \hbar T} \int_{0}^{T} d t_{1} \int_{t_{1}}^{T} d t_{2} C_{t_{2}}\left(\mathbf{p}\left(t_{1}\right), \mathbf{q}\left(t_{1}\right)\right) \\
& \times\left\langle\chi \mid \mathbf{p}\left(t_{2}\right), \mathbf{q}\left(t_{2}\right)\right\rangle e^{i\left(S_{t_{2}}(\mathbf{p}(0), \mathbf{q}(0))+E t_{2}\right) / \hbar}\left[\left\langle\chi \mid \mathbf{p}\left(t_{1}\right), \mathbf{q}\left(t_{1}\right)\right\rangle e^{\left.i\left(S_{t_{1}}(\mathbf{p}(0), \mathbf{q}(0))+E t_{1}\right) / \hbar\right]^{*}}\right.
\end{aligned}
$$

where $\left(\mathbf{p}\left(t_{1}\right), \mathbf{q}\left(t_{1}\right)\right)$ and $\left(\mathbf{p}\left(t_{2}\right), \mathbf{q}\left(t_{2}\right)\right)$ are variables that evolve from the same initial conditions but to different times, and $T$ is the total simulation time. The advantage of this approach is that the additional time integral can in principle replace the need for phase-space averaging in the large-time limit of a single trajectory. Calculations of the vibrational spectra of systems such as the water molecule have proved to be very accurate using the TA-SC-IVR approach and its inexpensive single-trajectory variant showed significant improvements over the simple harmonic approximation for excited vi- 
brational levels [26]. In order to make Eq. (4) less computationally demanding, one can employ the separable approximation [26], where the pre-factor of Eq. (4) is approximated as a phase, $C_{t_{2}}\left(\mathbf{p}\left(t_{1}\right), \mathbf{q}\left(t_{1}\right)\right)=\operatorname{Exp}\left[i\left(\phi\left(t_{2}\right)-\phi\left(t_{1}\right)\right) / \hbar\right]$, and $\phi(t)=$ phase $\left[C_{t}(\mathbf{p}(0), \mathbf{q}(0))\right]$. Using this approximation, Eq. (4) becomes

$$
\begin{aligned}
I(E) & =\frac{1}{(2 \pi \hbar)^{F}} \frac{1}{2 \pi \hbar T} \int d \mathbf{p}(0) \int d \mathbf{q}(0) \\
& \times \mid \int_{0}^{T} d t\langle\chi \mid \mathbf{p}(t), \mathbf{q}(t)\rangle \\
& \times\left. e^{i\left(S_{t}(\mathbf{p}(0), \mathbf{q}(0))+E t+\phi_{t}(\mathbf{p}(0), \mathbf{q}(0)) / \hbar\right)}\right|^{2}
\end{aligned}
$$

leading to a simplification of the double-time integration to a single time integral. The resulting integral is positive definite, making more amenable for Monte Carlo integration. Our numerical tests show that the results of carrying out this approximation are essentially identical to the double time integral approach when using a single trajectory. In this paper results will be reported by use of this last approximation, since it is computationally cheaper and numerically more stable than Eq. (4).

For this work, we compute the potential energy surface at each nuclear configuration directly from the Kohn-Sham orbitals expanded on a non-orthogonal Gaussian basis. Gradients and Hessians at each nuclear configuration are obtained analytically from electronic orbitals. The evaluation of the potential represents most of the computational effort of our approach, which is roughly few hours of computer time using standard desktop machines for a $1 \mathrm{~cm}^{-1}$ spectrum resolution. The nuclear equations of motion are

$$
M_{I} \ddot{\mathbf{R}}_{I}=-\nabla_{I} \min _{\mathbf{C}} E_{D F T}\left[\mathbf{C}, \mathbf{R}_{I}\right]
$$

where $\mathbf{C}$ is the rectangular matrix of the lowest occupied orbitals and the classical propagation is performed according to the velocity-Verlet algorithm, as implemented in the Q-Chem package [28]. At each time step, the potential, nuclear gradient and Hessian are used to calculate the action, pre-factor and coherent state overlaps necessary for the TA-SC-IVR method (Eqs. 4 and 5). A schematic representation of an implementation of the algorithm for a multithreaded machine is shown in Fig. (1). At each time step, results are accumulated for time-average integration. The results presented on this work were carried out on a single thread. For each classical trajectory, the procedure is repeated and the final integration gives the spectrum intensity $I(E)$ for a given parametric value of $E$. The same procedure is repeated for next $E+\Delta E$, where in our calculation $\Delta E=1 \mathrm{~cm}^{-1}$. As previously mentioned, the trajectory is monitored by calculating at each time step the deviation of the determinant of the monodromy matrix from unity. The difference in the determinants was always smaller than $10^{-6}$ during the course of the calculations. A time step of 10 a.u. has been always found to satisfy the strict monodromy matrix restrictions even for the lightest atoms.

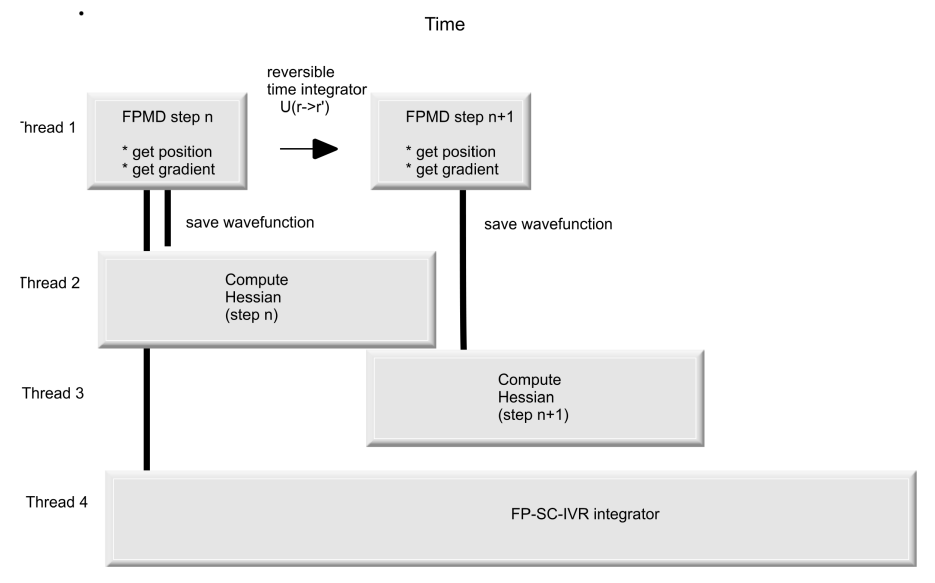

Figure 1: First-principles SC-IVR algorithm: At each time step electronic wavefunction are saved to calculated nuclear Hessian. Nuclear positions, gradients and Hessian are accumulated for the spectral time-average integral.

The calculation of the full dimensional vibrational power spectrum of the $\mathrm{CO}_{2}$ molecule is a challenging test for FP-SCIVR method: A successful method should reproduce spectral features such as degenerate bending modes, strong intermodal couplings and Fermi resonances. To evaluate the FP-SC-IVR method, we compare vibrational spectrum of $\mathrm{CO}_{2}$ molecule from FP-SC-IVR method to numerically-exact discrete variable representation (DVR) eigenvalue calculations on a potential fitted to a set of first-principles points obtained at the same level of theory. The next section describes the details of the potential fitting and DVR calculation. Following, we continue on the discussion of the FP-SC-IVR method.

\section{POTENTIAL FITTING AND GRID CALCULATIONS}

The $\mathrm{CO}_{2}$ molecule is a linear molecule with four vibrational normal modes: a symmetric stretching mode $\left(v_{1}\right)$, degenerate bending modes $\left(v_{2}\right.$ and $\left.\bar{v}_{2}\right)$, and an antisymmetric stretching mode $\left(v_{3}\right)$. A $3 d$ potential energy grid in internal coordinates is calculated using the B3LYP density functional [29] with the cc-pVDZ basis set [30]. The grid points are then fitted to a potential energy surface [31] represented by a fourth-order Morse-cosine expansion

$$
\begin{aligned}
V\left(r_{1}, r_{2}, \theta\right) & =\sum_{i, j, k=0}^{4} K_{i j k}\left(1-e^{-a_{1}\left(r_{1}-r_{e}\right)}\right)^{i} \\
& \times\left(\cos \theta-\cos \theta_{e}\right)^{j}\left(1-e^{-a_{2}\left(r_{2}-r_{e}\right)}\right)^{k}
\end{aligned}
$$

where the parameter $r_{e}=2.206119$ a.u. and $\theta_{e}=180$ specify the equilibrium coordinates of the $\mathrm{CO}_{2}$ molecule. The Morse parameters $a_{1}=a_{2}=1.2489$ a.u. were determined so as to minimize the standard deviation of the differences of the fitted potential from the ab initio result using the LevenbergMarquardt non-linear least square algorithm [32] . Instead, $r_{e}$ 


\begin{tabular}{cc||cc}
\hline coeff. & attoJ & coeff. & attoJ \\
\hline \hline$K_{001}$ & +0.000000 & $K_{100}$ & $=K_{001}$ \\
$K_{002}$ & +1.442886 & $K_{200}$ & $=K_{002}$ \\
$K_{003}$ & -0.032125 & $K_{300}$ & $=K_{003}$ \\
$K_{004}$ & +0.003630 & $K_{400}$ & $=K_{004}$ \\
$K_{010}$ & +0.726891 & $K_{111}$ & +0.392310 \\
$K_{011}$ & -0.443422 & $K_{110}$ & $=K_{011}$ \\
$K_{012}$ & -0.162970 & $K_{210}$ & $=K_{012}$ \\
$K_{013}$ & -0.101077 & $K_{310}$ & $=K_{013}$ \\
$K_{020}$ & +0.488451 & $K_{121}$ & +0.606572 \\
$K_{021}$ & -0.358126 & $K_{120}$ & $=K_{021}$ \\
$K_{022}$ & -0.210888 & $K_{220}$ & $=K_{022}$ \\
$K_{030}$ & +0.175981 & $K_{202}$ & +0.097300 \\
$K_{031}$ & -0.184503 & $K_{130}$ & $=K_{031}$ \\
$K_{112}$ & +0.103205 & $K_{211}$ & $=K_{112}$ \\
$K_{101}$ & +0.210532 & $K_{040}$ & +0.155374 \\
$K_{102}$ & +0.067998 & $K_{201}$ & $=K_{102}$ \\
$K_{103}$ & +0.068693 & $K_{301}$ & $=K_{103}$
\end{tabular}

Table I: Expansion coefficients $K_{i j k}$ for the $\mathrm{CO}_{2}$ B3LYP/cc-pVDZ fitted potential energy surface in attoJoule units.

was obtained by geometry optimization within the Q-Chem ab initio package [28].

The $35 K_{i j k}$ coefficients were subject to the non-linear least square fitting procedure to the DFT energies. Since these coefficients must be the same once $r_{1}$ and $r_{2}$ are swapped, 13 linear constraints of the type $K_{i j k}=K_{k j i}$ were imposed during the fitting procedure. Additionally, to ensure that the equilibrium geometry was fitted to the predetermined equilibrium parametric distance, the coefficients $K_{100}$ and $K_{001}$ were constrained to be zero. Consequently, we employed a total number of 14 fitting constraints ( $K_{000}$ term is always constant). A total of $2500 \mathrm{ab}$ initio grid points were chosen for the fitting process. These grid points range from 1.42 a.u. to 7.09 a.u. for $r_{1}$ and $r_{2}$, and from 113.6 to 180 for the angle variable. The calculated expansion coefficients $K_{i j k}$ are reported in Tab.(I).

As far as the numerically exact eigenvalues calculations is concerned, we used an exact DVR (Discrete Variable Representation) matrix diagonalization procedure. The $\mathrm{CO}_{2}$ molecule was described for grid calculations in internal coordinates, while on-the-fly classical trajectories and the semiclassical calculations described previously were performed in Cartesian coordinates. No significant contamination between the rotational (set to zero kinetic energy) and vibrational motion was found within the simulation time. To this end, the deviation from simplecticity of the monodromin matrix in the vibrational sub-space were never more than $10^{-6}$ as previously mentioned.

The coordinates $r_{1}$ and $r_{2}$ are $\mathrm{CO}$ distances, and $\theta$ is the angle between the $\mathrm{CO}$ bonds. In these coordinates the kinetic part of the Hamiltonian for $J=0$ is

$$
\begin{aligned}
T= & \frac{p_{1}^{2}}{2 \mu_{C O}}+\frac{p_{2}^{2}}{2 \mu_{C O}}+\frac{j^{2}}{2 \mu_{C O} r_{1}^{2}}+\frac{j^{2}}{2 \mu_{C O} r_{2}^{2}}+\frac{p_{1} p_{2} \cos \theta}{m_{C}} \\
& -\frac{p_{1} p_{\theta}}{m_{C} r_{2}}-\frac{p_{2} p_{\theta}}{m_{C} r_{2}}-\frac{\cos \theta j^{2}+j^{2} \cos \theta}{2 m_{C} r_{1} r_{2}}
\end{aligned}
$$

where

$$
\begin{gathered}
p_{k}=-i \frac{\partial}{\partial r_{k}}, k=1,2 \\
p_{\theta}=-i \frac{\partial}{\partial \theta} \sin \theta
\end{gathered}
$$

and

$$
j^{2}=-\frac{1}{\sin \theta} \frac{\partial}{\partial \theta} \sin \theta \frac{\partial}{\partial \theta}
$$

The carbon mass were taken to be $m_{C}=12.0$ a.m.u., while the oxygen mass $m_{O}=15.9949$ a.m.u. and the reduced mass is as usual $1 / \mu_{C O}=1 / m_{C}+1 / m_{O}$.

As previosuly mentioned, in order to calculate exact eigenvalues, a sine-DVR basis for the coordinates $r_{1}$ and $r_{2}$ and a Legendre-DVR basis for $\theta$ has been used [33]. For each degree of freedom 50 DVR functions were used and eigenvalues were converged to at least $10^{-3} \mathrm{~cm}^{-1}$. The sine-DVR ranged from 1.51 a.u. to 3.78 a.u. and the magnetic quantum number $m$ of the Legendre-DVR was zero.

Because of the restriction of total angular momentum $J=0$, we couldn't observe all degenerate bending excitations. However, ZPE and several vibrational energy levels were obtained and compared with that ones coming from a single on-the-fly semiclassical trajectory.

\section{FIRST-PRINCIPLES SC-IVR CALCULATIONS}

The full power spectrum obtained using Eq. (4) after 3000 BOMD steps of 10 a.u. each is shown on the bottom of Fig. 2. For longer simulations, the monodromy matrix symplectic properties as well as the resolution of the spectrum started to deteriorate. The calculated vibrational zero-point energy (ZPE) value was $2518 \mathrm{~cm}^{-1}$ versus the exact value of $2514.27 \mathrm{~cm}^{-1}$ and both are in good agreement with the experimental value of $2508 \mathrm{~cm}^{-1}$. In contrast, harmonic normal-mode analysis (whose frequencies are $656.62,1363.46,2423.47$ wavenumbers) predicts a frequency of $2550.08 \mathrm{~cm}^{-1}$. Thus, the TA-SC-IVR method successfully reproduces the ZPE anharmonic effects with the use of a single classical trajectory. Some representative frequencies of the power spectrum are presented in Table II. The ZPE was shifted to zero for comparison with reported classical ELMD simulations on the same system that cannot reproduce the ZPE or higher vibrational states $[34,35]$ but only single modes frequencies. For these studies of Refs. [34, 35], the vibrational 


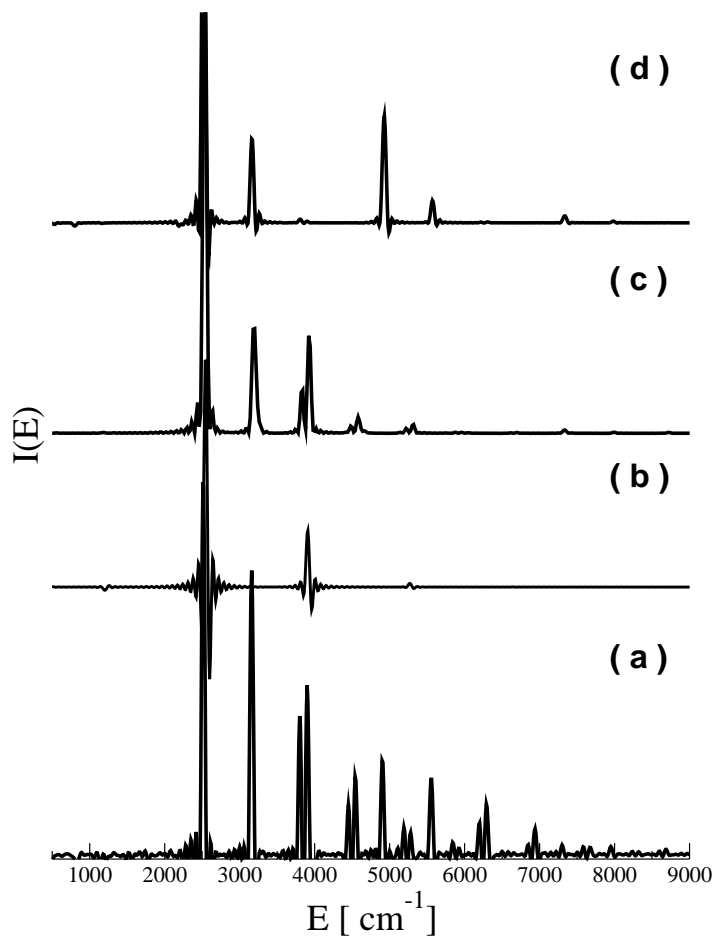

Figure 2: $\mathrm{CO}_{2}$ Vibrational Power Spectrum: Initial kinetic energy on: (a) all modes; (b) symmetric mode; (c) one bending and symmetric modes; (d) bending and asymmetric modes.

data were obtained from the Fourier transform of correlation functions of classical trajectories in plane-wave DFT calculations. The ELMD approach predicts the following fundamental frequencies 648, 1368, 1428 and 2353 for Ref. [34] and 663, 1379, 1456 and 2355 for Ref. [35]. These classical results are similar but limited to a normal mode analysis.

Table II compares our TA-SC-IVR results with the exact ones and to those obtained by Filho [36] with the same density functional and a basis set of comparable quality $\left(6-31+\mathrm{G}^{*}\right)$ [37], using a perturbative approximation of the eigenvalue expansion. One can see how a different basis set results a significant deviation of vibrational levels spacing, once the comparison is performed in units of wavenumbers.

A major difficult on the $\mathrm{CO}_{2}$ power spectrum simulations is the calculation of the Fermi resonance splittings. These are the result of anharmonic couplings, and they represent a stringent test for a semi-classical method that relies on a single short trajectory. The Fermi resonances occur when an accidental degeneracy between two excited vibrational levels of the same symmetry exists and it results in a repulsion between the corresponding energy levels. The sources of these resonances are purely anharmonic and are only present in polyatomic potentials. For the $\mathrm{CO}_{2}$ molecule, the unperturbed frequencies for the symmetric stretching are roughly equal to the first bending overtone $\left(v_{1} \cong 2 v_{2}\right)$. For these modes, the wavefunctions are transformed as the irreducible representation of $D_{\infty h}$, i.e. $v_{1}\left(10^{0} 0\right)$ as $\Sigma_{g}^{+}$, at the experimental frequency of $1388 \mathrm{~cm}^{-1}$, and $v_{2}^{2}\left(02^{0} 0\right)$ as $\Sigma_{g}^{+}+\Delta_{g}$, at an experimental fre-

\begin{tabular}{rccccc}
\hline Exp. $^{a}$ & mode $^{b}$ & Harmonic $^{c}$ & FP-SCIVR-SA $^{d}$ & DVR & Ref. [36] \\
\hline \hline 667.4 & $0,1^{1}, 0$ & 656.62 & 644 & & 657.2 \\
$1285.4^{\wedge}$ & $0,2^{0}, 0$ & 1313.24 & 1288 & 1252.91 & 1283.4 \\
$1388.2^{\wedge}$ & $1,0^{0}, 0$ & 1363.46 & 1381 & 1372.29 & 1408.8 \\
$1932.5^{\dagger}$ & $0,3^{1}, 0$ & 1969.86 & 1932 & & 1930.2 \\
2003.2 & $0,3^{3}, 0$ & 1969.86 & 2024 & & 2004.9 \\
$2076.9^{\dagger}$ & $1,1^{1}, 0$ & 2020.08 & 2106 & & 2098.5 \\
2349.1 & $0,0^{0}, 1$ & 2423.47 & 2388 & 2359.51 & 2411.5 \\
$2548.4^{\ddagger}$ & $0,4^{0}, 0$ & 2626.48 & 2515 & 2482.95 & 2553.3 \\
$2585.0^{\star}$ & $0,4^{2}, 0$ & 2626.48 & 2578 & & 2591.2 \\
$2671.7^{\ddagger}$ & $0,4^{4}, 0$ & 2626.48 & 2669 & 2640.15 & 2716.5 \\
$2760.7^{\star}$ & $1,2^{2}, 0$ & 2676.70 & 2759 & & 2796.3 \\
$2797.2^{\ddagger}$ & $2,0^{0}, 0$ & 2726.92 & 2793 & 2757.14 & 2845.2 \\
4673.3 & $0,0^{0}, 2$ & 4846.94 & $4690^{+}$ & 4693.24 & 4797.8 \\
6972.6 & $0,0^{0}, 3$ & 7270.41 & $6803^{+}$ & 6821.35 & 7152.9 \\
\hline
\end{tabular}

${ }^{a}$ Experimental frequencies in $\mathrm{cm}^{-1}$ from Ref. [41]

${ }^{b}$ First number is the symmetric stretch quantum, second are the degenerate bendings, and third one is the asymmetric stretch. The exponent of the second number is the $l_{i}$ degeneracy index.

${ }^{c}$ Vibrational levels according to a normal modes harmonic model

${ }^{d}$ Using the Separable approximation of Eq.(5)

Table II: Some of the calculated vibrational energy eigenvalues. All data are in wavenumbers. Fermi Resonances group of frequencies are indicated by the same superscript symbols. Uncertain peaks are marked with $(+)$. The first column represents the experimental vibrational frequencies associated with the modes listed on the second column. The third column shows the harmonic DFT results. In the fourth and fifth columns, we show our FP-SCIVR and exact numerical DVR calculations in the B3LYP/cc-PVDZ model chemistry used for the FP-SCIVR calculations. The fifth column shows perturbative DFT calculations carried out using a similar functional and basis set.

quency of $1285 \mathrm{~cm}^{-1}$. Another Fermi doublet results from the addition of a quantum of bending mode to the previous Fermi doublet to yield the following states: $v_{1} v_{2}\left(11^{1} 0\right)$, at an experimental frequency of $2077 \mathrm{~cm}^{-1}$ and the $v_{2}^{3}\left(03^{1} 0\right)$ state, at an experimental frequency of $1932 \mathrm{~cm}^{-1}$. Higher-energy Fermi resonances are indicated in Table II by using the same superscript symbols. The first Fermi terms are located at 1313 and 1363 in a harmonic approximation and corrected to 1288 and 1381 wavenumbers for FP-TA-SC-IVR. Thus, the original levels have been repelled by Fermi couplings. One mode is located at a higher frequency than the harmonic prediction, while the other is at a lower frequency. The latter effect could be explained also by simple anharmonicity, but the former is evidence of the ability of the single trajectory FP-TA-SCIVR method even when the separable approximation is used to capture Fermi resonance effects partially. The same reasoning can explain the second Fermi doublet located at 1932 and 2106 for FP-TA-SC-IVR, while the harmonic estimate at 1970 and 2020 wavenumbers.

With the FP-TA-SC-IVR method, one can also identify the couplings between vibrational modes and the appearance of Fermi resonance splittings by carrying out simulations with different initial conditions. This can be achieved by selec- 
tively setting the initial velocity of some vibrational modes to zero. The anharmonic coupling between levels leads to a consistent reproduction of the ZPE peak in the spectrum for all simulations. However the excited vibrational peaks related to the modes with zero initial kinetic energy show a very small signal in the power spectrum. Vibrational energy redistribution processes can be studied as well, by carrying out simulations at different timescales. In Fig. 2, we show the resulting power spectra for different initial conditions. If the initial state contains only purely symmetric motion, the lowest Fermi resonance peaks in Fig. 2(b) are absent as well as for a bending (without symmetric stretching) motion in Fig. 2(d). These results and the intensity of their peaks respect to that ones located at the same frequencies in Fig. 2(a) suggest that the Fermi resonance is indeed originated from the coupling between bending and the symmetric modes. One can reach the same conclusions by inspecting the lower Fermi doublet peaks intensity: by adding a bending mode (from Fig. 2(b) to Fig. 2(c)) and a second one (from Fig. 2(c) to Fig. 2(a)) the intensity of both peaks is gradually raised. This is called "intensity borrowing" and it arises from the strong mixing of the zero order states. These observations reinstate that "repulsion and mixing are the hallmarks of Fermi resonances" [38]. Also, for a distinct set of initial conditions, an additional peak at 5500 $\mathrm{cm}^{-1}$ related to the asymmetric stretch was observed. Using the proposed approach, one can carefully detect the characteristics of each peak even for complicated power spectra.

An attractive method for obtaining the symmetry properties of the eigenstates involves arranging the initial basis vectors $[26,39]$. The basis for this method is the direct product of coherent states $|\chi\rangle=\prod_{k=1}^{4}\left|p_{i}^{(k)}, q_{i}^{(k)}\right\rangle^{\varepsilon_{k}}$. These states can be chosen to have an initial symmetry by employing linear combinations of the form $\left|p_{i}^{(k)}, q_{i}^{(k)}\right\rangle^{\varepsilon_{k}}=$ $\left(\left|p_{i}^{(k)}, q_{i}^{(k)}\right\rangle+\varepsilon_{k}\left|-p,-q_{i}^{(k)}\right\rangle\right) / \sqrt{2}$. The $k$-th mode can be made symmetric $\left(\varepsilon_{k}=1\right)$, antisymmetric $\left(\varepsilon_{k}=-1\right)$ or have no symmetry restrictions $\left(\varepsilon_{k}=0\right)$. In order to assign the proper symmetry to each peak on Fig. 3 , the reduced $D_{2 h}$ symmetry group was adopted. All irreducible representations were reproduced and peaks were grouped by symmetry as reported in Fig. 3. Note that (d) and (e) plots are identical since they only differ trivially by swapping coefficients between the degenerate bending modes in the original $D_{\infty h}$ symmetry group.

Finally we have investigated the stability of the propagator versus variations of the coherent states gaussian width parameters $\gamma_{i}$. Previous calculations [24] have shown that there is no significant depedency on energy and norm conservation for the semiclassical propagator if suitable values of $\gamma_{i}$ are chosen. For power spectra calculation we have chosen to look at vibrational levels variations under different values of coeherent states width. Since a single trajectory was used in the FP-TA-SC-IVR approach, no Monte Carlo integration is performed in phase space coordinates and the changes of $\gamma_{i}$ are confined to the coherent states overlap and to the prefactor in Eq. (2). As reported in Fig. 4 and checked on a finer scale, no significant variation was observed beyond $1 \mathrm{~cm}^{-1}$. These find-

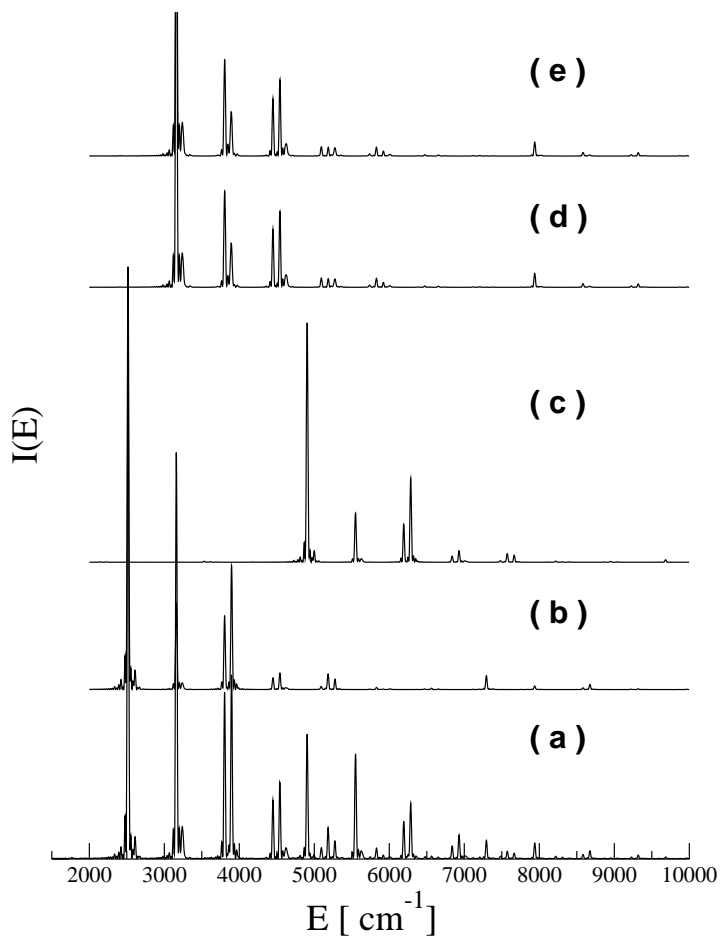

Figure 3: $\mathrm{CO}_{2}$ Vibrational Power Spectrum (Separable approximation): Different basis set symmetries for $v_{1}$ (symmetric stretching mode), $v_{2}$ and $\bar{v}_{2}$ (bending modes) and $v_{3}$ (asymmetric mode) and the corresponding $D_{2 h}$ irreducible representation; (a) all $\varepsilon$ s are zero; (b) $\left(B_{1 u}\right): \varepsilon\left(v_{1}\right)=0, \varepsilon\left(v_{2}\right)=1, \varepsilon\left(\bar{v}_{2}\right)=0, \varepsilon\left(v_{3}\right)=-1$; (c) $\left(A_{g}\right): \varepsilon\left(v_{1}\right)=1, \varepsilon\left(v_{2}\right)=0, \varepsilon\left(\bar{v}_{2}\right)=0, \varepsilon\left(v_{3}\right)=1 ;(\mathrm{d})\left(B_{2 u}\right): \varepsilon\left(v_{1}\right)=$ $0, \boldsymbol{\varepsilon}\left(v_{2}\right)=-1, \varepsilon\left(\bar{v}_{2}\right)=0, \varepsilon\left(v_{3}\right)=1$, (e) $\left(B_{3 u}\right) \varepsilon\left(v_{1}\right)=0, \varepsilon\left(v_{2}\right)=$ $0, \varepsilon\left(\bar{v}_{2}\right)=-1, \varepsilon\left(v_{3}\right)=1$. $B_{2 u}$ and $B_{3 u}$ representations are degenerated in the $D_{\infty h}$ subspace as shown.

ings are in agreements with previous calculations on the same propagator [24]. Interestingly, a different distribution in peaks intensity were found in each panel. Since the peaks magnitude is proportional to the overlap between the reference state and the actual eigenfunction, the anharmonic choice $\left(\gamma_{i}=\omega_{i} / 2\right)$ is a more suitable solution as clearly showed on panel (c) of Fig. 4.

\section{CONCLUSIONS}

In conclusion, we have shown that SC-IVR can be implemented easily and efficiently using first principles molecular dynamics. With the modest computational cost of a single classical trajectory, the vibrational density of states of the $\mathrm{CO}_{2}$ molecule was calculated. On Fig. 5 we report a graphical comparison between the harmonic and the FP-TA-SC-IVR approximations, versus the exact vibrational value for the Fermi resonance multiplets. One can notice how the single trajectory FP-TA-SC-IVR goes far beyond the harmonic approximation by removing the harmonic degenerancy and including part of anharmonicity. Fermi splittings are well mimiced not only for the first doublet, but also for the higher ones. The 


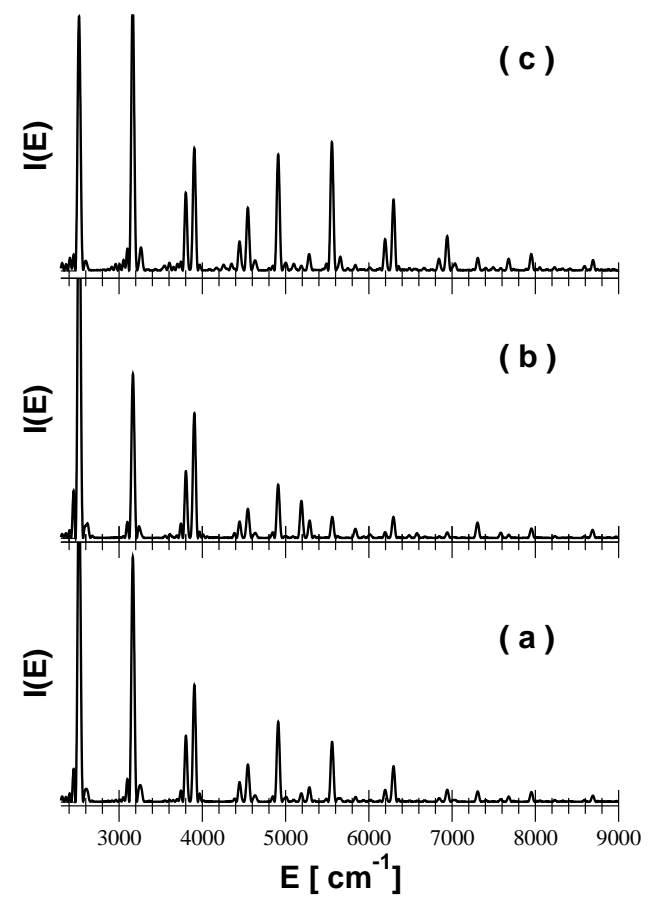

Figure 4: Gaussian width variations and related power spectra: a) $\gamma_{i}=\omega_{i}$; b) $\gamma_{i}=2 \omega_{i}$; c) $\gamma_{i}=\omega_{i} / 2$, where $\omega_{i}$ are the $i$-esime normal mode frequency. The FP-SCIVR power spectra are fairly insensitive to variations in the value of the coherent state width.

numerically exact DVR vibrational energy levels constrained by $J=0$ are represented on the last column. The FP-TASC-IVR values are similar to the DVR results, when comparison is possible. However, a closer look at Table (II) shows how these single trajectory FP-TA-SC-IVR calculations can include only part of the anharmonicity and that their precision gets worse for higher vibrational levels. In particular, the spacing of the higher-energy states is harmonic-like and thisis the mayor limitation of using a single classical trajectory.

These and previous calculations on model potentials [26] has shown how the single trajectory TA-SC-IVR gives reasonable results and performs better for higher frequencies modes. The computational cost of the method is essentially the same as classical propagation, and therefore, if broadly implemented in electronic structure codes, it can provide a description of quantum effects at a comparable computational cost to that of classical approaches. Possible applications of this method or related ones are the study of excited electronic states and Franck-Condon transitions, such as vibrational absorption spectra [42]. Although this single trajectory approach may be a practical tool for the simulation of more complex systems, the use of more trajectories is probably required to remove any harmonic "ghost states". We are currently exploring the use of a small number of a set of systematically determined trajectories for further improvement of the results. If the number of required trajectories grows as a low polynomial of the system size, semi-classical methods could be competitive with currently-employed numerical approximations to (a) (b) (c)

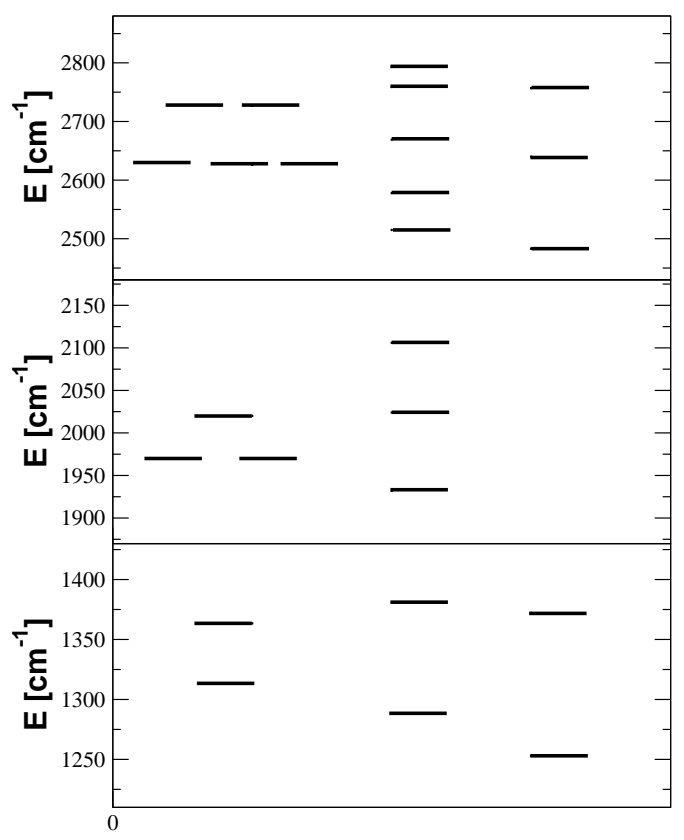

Figure 5: Fermi Resonance states vibrational energy level: (a) in harmonic approximation; (b) single FP-SC-IVR trajectory calculation; (c) exact grid calculation on splined potential.

obtain anharmonic vibrational effects. Finally, we expect that the representation of the potential energy in terms of normal coordinates will become less suitable when large amplitude motions or non adiabatic effects come into play.

\section{Acknowledgement}

One of the authors (M.C.) feels deeply in debt with Prof. W. $\mathrm{H}$. Miller for the many lessons learned from him. The authors thank Dr. A. Kaledin, Prof. E. J. Heller for useful discussions and revision of the manuscript. A. A.-G., S. S. and S. A. thank the Faculty of Arts and Sciences of Harvard University for financial support and S. S. thanks the Samsung Scholarship for financial support. M. C. and G. F. T. thanks the University of Milan for fundings and CILEA (Consorzio Interuniversitario Lombardo per L'Elaborazione Automatica) for computational time allocation. A. A.-G., S. S. and S.A thank FAS Research Computing for cluster computing support.

$\dagger$ Electronic address: michele.ceotto@unimi.it, aspuru@ chemistry. harvard.edu

[1] J. M. Herbert and M. Head-Gordon, Phys. Chem. Chem. Phys., 2005, 7, 3269.

[2] R. Car and M. Parrinello, Phys. Rev. Lett., 1985, 55, 2471.

[3] H. B. Schlegel, J. M. Millam, S. S. Iyengar, G. A. Voth, A. D. Daniels, G. E. Scuseria, and M. J. Frisch, J. Chem. Phys., 2001, 
114, 9758 .

[4] J. M. Herbert and M. Head-Gordon, J. Chem. Phys., 2004, 121, 11542.

[5] Y. Liu, D. Yarne, M. E. Tuckerman, Phys. Rev. B, 2003, 68, 125110.

[6] P. Tangney, J. Chem. Phys., 2006, 124, 044111.

[7] M. Pavese, D. R. Berard, G. A. Voth, Chem. Phys. Lett., 1999, 300, 93.

[8] G. A. Worth, M. A. Robb and I. Burghardt, Faraday Discuss., 2004, 127, 307.

[9] S. Iyengar and J. Jakowski, J. Chem. Phys., 2005, 122, 114105.

[10] O. Knospe and P. Jungwirth, Chem. Phys. Lett., 2000, 317, 529.

[11] W. H. Miller, Adv. Chem. Phys., 1974, 25, 69; W. H. Miller, Faraday Discuss., 1998, 110, 1.

[12] W. H. Miller, J. Chem. Phys., 1970, 53, 3578; ibidem, 1970, 53, 1949; W. H. Miller, J. Phys. Chem. A, 2001, 105, 2942; M. Thoss and H. Wang, Annu. Rev. Phys. Chem., 2004, 55, 299; K. G. Kay, Annu. Rev. Phys. Chem., 2005, 56, 255.

[13] H. Wang, X. Sun, and W. H. Miller, J. Chem. Phys., 1988, 108, 9726; X. Sun and W. H. Miller, J. Chem. Phys., 1999, 110, 6635; M. Thoss, H. Wang and W. H. Miller, J. Chem. Phys., 2001, 114, 9220; T. Yamamoto, H. Wang, and W. H. Miller, J. Chem. Phys., 2002, 116, 7335; T. Yamamoto, W. H. Miller, J. Chem. Phys., 2003, 118, 2135.

[14] J. Ankerhold, M. Saltzer, and E. Pollak, J. Chem. Phys., 2002, 116, 5925; S. Zhang and E. Pollak, Phys. Rev. Lett., 2003, 91, 190201; S. S. Zhang and E. Pollak, J. Chem. Phys., 2004, 121, 3384.

[15] A. R. Walton, D. E. Manolopoulos, Mol. Phys., 1996, 87, 961; A. R: Walton, D. E. Manolopoulos, Chem. Phys. Lett., 1995, 244, 448; M. L. Brewer, J. S. Hulme, D. E. Manolopoulos, J. Chem. Phys., 1997, 106, 4832.

[16] S. Bonella, D. Montemayor, and D. F. Coker, Proc. Natl. Am. Soc., 2005, 102, 6715; S. Bonella and D. F. Coker, J. Chem. Phys., 2003, 118, 4370.

[17] Y. Wu , M. Herman, V. S. Batista, J. Chem. Phys., 2005, 122, 114114; Y. Wu and V. S. Batista, J. Chem. Phys., 2003, 118, 6720.

[18] F. Grossmann, Comments At. Mol. Phys., 1999, 34, 243.

[19] E. J. Heller, J. Chem. Phys., 1975, 62, 1544; E. J. Heller, J. Chem. Phys., 1981, 75, 2923.

[20] E. J. Heller, Acc. Chem. Res., 1981, 14, 368; E. J. Heller, Acc. Chem. Res., 2006, 39, 127.

[21] T. Van Voorhis, and E. J. Heller, J. Chem. Phys. , 2003, 119, 12153

[22] D. V. Shalashilin and M. S. Child, Chem. Phys., 2004, 304,
103; D. V. Shalashilin and M. S. Child, J. Chem Phys., 2001, 115, 5367.

[23] M. Ben-Nun, T. J. Martinez, Adv. Chem. Phys., 2002, 121, 439.

[24] M. F. Herman and E. Kluk, Chem. Phys., 1984, 91, 27; K. G. Kay, J. Chem. Phys., 1994, 100, 4377; K. G. Kay, J. Chem. Phys., 1994, 100, 4432.

[25] H. Wang, D. E. Manolopoulos, and W. H. Miller, J. Chem. Phys., 2001, 115, 6317.

[26] A. L. Kaledin and W. H. Miller, J. Chem. Phys., 2003, 118, 7174; M. Ceotto, PhD Dissertation, University of California, Berkeley (2005); A. L. Kaledin and W. H. Miller, J. Chem. Phys., 2003, 119, 3078.

[27] Y. Elran and K. G. Kay, J. Chem. Phys., 1999, 110, 3653; ibidem, 1999, 110, 8912.

[28] Y. Shao, et al. Phys. Chem. Chem. Phys., 2006, 8, 3172.

[29] A.D. Becke, J. Chem. Phys., 1993, 98, 5648; P. J. Stephens, F. J. Devlin, C. F. Chabalowski, and M. J. Frisch, J. Phys. Chem., 1994, 98, 11623.

[30] T. Dunning, Jr. J. Chem. Phys., 1989, 90, 1007.

[31] J. Zuniga, M. Alacid, A. Bastida, F. J. Carvajal, and A. Requena, J. Mol. Spectr., 1999, 195, 137.

[32] K. Levenberg, Quart. Appl. Math. 2, 164 (1944); D. Marquardt, Siam J. Appl. Math. 11, 431 (1965); M.I.A. Lourakis, Levenberg-Marquardt nonlinear least squares algorithms in C/C++, http://www.ics.forth.gr/ lourakis/levmar/ (2004)

[33] M. H. Beck and H.-D. Meyer, J. Chem. Phys., 2001, 114, 2036; G. A. Worth, M. H. Beck, A. Jäckle, and H.-D. Meyer, The MCTDH Package, Version 8.2, (2000), University of Heidelberg, Heidelberg, Germany, H.-D. Meyer, Version 8.3 (2002). See http://www.pci.uni-heidelberg.de/tc/usr/mctdh/

[34] F. Gygi, Phys. Rev. B, 1995, 51, 11190.

[35] J. R. Chelikowsky, X. Jing, K. Wu, and Y. Saad, Phys. Rev. B, 1994, 53, 12071.

[36] H. P. M. Filho, Spectr. Acta Part A, 2002, 58, 2621.

[37] W. J. Hehre, R. Ditchfield and J. A. Pople, J. Chem. Phys., 1972, 56, 2257.

[38] E. J. Heller, E. B. Stechel, and M. J. Davis, J. Chem. Phys., $1980,73,4720$.

[39] X. Sun and W. H. Miller, J. Chem. Phys., 1998, 108, 8870.

[40] A. M. N. Niklasson, C. J. Tymczak, and M. Challacombe, Phys. Rev. Lett., 2006, 97, 123001; A. M. N. Niklasson, C. J. Tymczak, and M. Challacombe, J. Chem. Phys., 2007, 126, 144103.

[41] L. R. Brown and C. B. Farmer, Appl. Opt., 1987, 26, 5154.

[42] J. Tatchen and E. Pollak, J. Chem. Phys., 2009, 130, 041103. 